Histopathology revealed an increased cell density of oligodendrocytes $\left(3350.5\right.$ cells $/ \mathrm{mm}^{2}$, compared to 1683.6 cells $/ \mathrm{mm}^{2}$ in adjacent normal-appearing white matter, $\mathrm{p}<0.001)$. Myelination (luxol fast blue staining) was normal. Supernumerary cells were negative for IDH1R132H by immunohistochemistry, IDH1/ 2 wildtype by Sequenom, and negative for Ki-67, synaptophysin, and BRAF V600E. The overlying cortex was completely normal, with no gliosis, balloon cell, neuronal dysmorophology or calcification. The original neuropathologic diagnosis was oligodendroglial hamartoma, although a more descriptive diagnosis of focal oligodendroglial hyperplasia is preferred. The patient continues to have 1-2 nocturnal partial (focal with dyscognitive features) seizures per month, but has had no convulsive seizure since her surgery (4.5 year follow-up). Oligodendroglial hyperplasia is a poorly characterized subtype of microdysgenesis, and a rare cause of medically refractory epilepsy. Histologically, it is important that it is differentiated from oligodendroglioma. Because of the paucity of published cases, the clinical impact of surgical resection of oligodendroglial hyperplasia found within the seizure onset zone is uncertain.

CONFlicts OF INTEREST:

None.

\section{Abstract A10}

\section{Synaptic plexi of heterotopic white matter neurons in epileptogenic focal cortical dysplasias}

Harvey B. Sarnat, Walter Hader, Laura Flores-Sarnat, Luis Bello-Espinosa

University of Calgary and Alberta Children's Hospital, Calgary, Alberta, Canada

\section{doi:10.1017/cjn.2015.376}

The objective was to define the role of excessive heterotopic white matter neurons beneath focal cortical dysplasias (FCD) for their contribution to epileptic circuitry. Synaptic plexi can be identified by synaptophysin immunoreactivity. Other neuronal markers can distinguish the origin of these neurons from the fetal subplate zone or by radial migration by using calretinin reactivity for GABAergic inhibitory interneurons or lack of calretinin expression by radial migratory excitatory neurons. Synaptophysin, calretinin and NeuN antibodies were applied to paraffin sections of surgical resections of epileptic foci in 16 infants, children and adolescents, ages 3 months to 17 years, with focus on subcortical axonal networks: 10 FCD I and 6 FCD II. Ten normal fetal brains of 18-22 gestational weeks, 6 of 37-41 weeks and 8 postnatal brains of infants and children at autopsy, and also white matter beneath normal cortex of surgical cases provided controls.

Subcortical and deep white matter in FCD exhibited excessive neurons, mostly scattered, but focally clustered and mainly at the depths of sulci. White matter neurons were reactive for NeuN and MAP2, but rarely for calretinin. Elaborate axonal plexi showed connections between heterotopic neurons and also with overlying cortex. Axons were oriented randomly and frequently projected to and entered the cortex; subcortical axons of controls ran parallel to cortex. In the grey matter of
FCD Ia and some cases of FCD II, radial layers of synapses alternated with micro-columns of neurons. The significance is that subcortical white matter neurons in FCD have elaborate synaptic plexi that probably contribute to epileptic circuitry. They are not derived from the subplate zone, hence contribute to excitatory rather than inhibitory networks. They also help explain indistinct grey/white MRI boundaries in FCD.

CONFLicts OF INTEREST:

None.

\section{Abstract A11}

\section{Shaken Baby Syndrome - Unravelled}

Roland N. Auer

University of Saskatchewan, Canada

doi:10.1017/cjn.2015.377

A generation of doctors has been taught that Shaken Baby Syndrome (SBS) can be diagnosed definitively by a triad of [1] retinal hemorrhage, [2] meningeal hemorrhage (subdural or subarachnoid) and [3] encephalopathy. SBS, not really syndromic, is unravelling, and has been supplanted by Abusive Head Trauma (AHT) by its proponents. Yet fundamental problems remain with the idea. The physics of angular acceleration provides only $11-12 \%$ of that necessary to cause destructive shear force within the brain. The fulcrum around which the brain would rotate is not the center of the head (as in boxing), but inferiorly in the neck, which has been injured in no case of presumptive SBS/AHT. Commonly, there is little to no differential diagnosis by child abuse specialists in court asserting there is only one possible diagnosis: abuse. There are a multitude of causes of the triad. Reperfusion hemorrhage after resuscitation following cardiac arrest accounts for many cases, due to reperfusion of previously hypoxic or ischemic endothelium. SBS and SIDS show a remarkable graphic superposition in month-over-month incidence plots, suggesting resuscitation in SIDS may turn it into SBS if reperfusion hemorrhage occurs. Another mimic of what passes for SBS/AHT is a short fall. Usually innocuous, short falls have a strike velocity of $>5 \mathrm{mph}$ from a 12 " fall, and $10 \mathrm{mph}$ from a 44 " fall and can give rise to fractures and/or subdural hematomas, if the head strikes first. Lucid intervals can occur in young children, but are discounted in SBS/AHT circles. Retinal hemorrhages result from $\uparrow I C P$ via the valveless venous system transmitting the pressure from the cerebral venous system to retinal veins. Differential diagnosis in law is termed due process, often lacking in SBS/AT cases at trial. The problem is confounded further by false confessions. Research on normal subjects shows that, if given cogent background data and a medical certainty they were guilty, people will admit to a factitious crime they never committed. These multiple, system-wide errors converge to cause incarceration of parents, day care workers and grandparents, presently numbering over 1000 imprisonments. Prosecutions based on the triad continue.

\section{CONFLICTS OF INTEREST:}

None. 